\title{
A Photometric Method for Following Changes in Length of Bacteria
}

\author{
By F. (O. POWHI.I AND P. J. STOWARD* \\ The Microbiological Research Establishment, Porton Down, Salisbury, Wiltshire
}

(Received 19 July 1961)

\begin{abstract}
SUMMARY
Rod-shaped particles suspended in a liquid undergo a partial orientation during flow; the flowing suspension has the property of seattering light mainly in certain preferred directions. The elementary theory of the phenomenon shows that it can be applied practically as a photometric method of following changes in length. A simple and effective apparatus for this purpose is described. and its performance illustrated. A brief discussion of size changes in synchronized cultures indicates the relation between these changes and the rate of fission.
\end{abstract}

\section{INTRODUCTION}

Progress in the study of synchirony in bacterial cultures has been handicapped in the past by the absence of a rapid and convenient means of measuring the concentration (by number) of a suspension. Conventional total or viable counts are slow and tedious, and do not meet the need for an immediate check on the occurrence of synchronous fission, and so on the efticacy of a trial procedure for inducing synchrony. Even a quite rough measurement would be acceptable, as indieating the occasions on which it would be profitable to carry out more exact counts. Instruments like the 'Coulter Counter', which enumerates the number of particles in a minute volume of suspension, have not yet been developed to the point at which they are both reliable and easy to use with bacterial suspensions.

As a method of monitoring if not of actual estimation, some form of photometric measurement would be especially convenient, but it is not to be expected that any standard instrument would be directly applicable. The turbidity of a suspension depends radically on both the size and concentration of the particles in it (other things being equal), and its true optical density bears no simple relation to these variables. In addition any conventional instrument intended as an absorptiometer gives, when used with suspensions, estimates of an apparent optical density which differs grossly from the truc one and which is a function of the geometry of the instrument (for cxamples, see Powell, 1962). It is often supposed that turbidity measurements correspond with concentrations of orranisms by mass, and this is roughly and empirically true of certain combinations of instrument and suspension, but there are many recorded examples to the contrary, in particular the interesting experiment of Stárka \& Koza (1959) discussed below.

In well-developed synchronous growth, there is no indication of any large lluctuation in the mass growth rate, but during periods of fission, the number concentra-

- Present address : Department of Human Anatomy, South Parks Road, Oxford. 
tion is nearly doubled and the mean length of the organisms is nearly halved. These two changes compensate one another to some extent so far as the light seattering of bulk suspensions is concerned. While it is possible in principle to determine the size and shape of suspended particles from their scattering propcrties, the measurements required are somewhat claborate in the most nearly idcal cases. Bacteria, differing in size and shape among themselves and not optically homogeneous, are quite intractable theorctically.

In this paper we describe a simple form of photonetric mcasurement which gives a direct indication of the axial ratio of rod-shaped particles and is almost independent of their concentration by number. It is thus particularly suitable for detecting the change in length which occurs during periods of fission in a synchronized culture and for this purpose no calibration is really necessary. The method is based on two phenomena: (i) non-spherical particles scutter light in ecrtain preferred directions which depend on their attitude relative to the direction of the incident light; (ii) in the laminar flow of a suspension, non-spherical particles tend to take up certain preferred orientations wherever there is a velocity gradient normal to the direction of llow. These phenomena are manifested in the familiar nacreous appearance of stirred suspensions of rod-shaped organisms, and in the fluctuating readings given by absorptiometers with such suspensions when they have not come to rest.

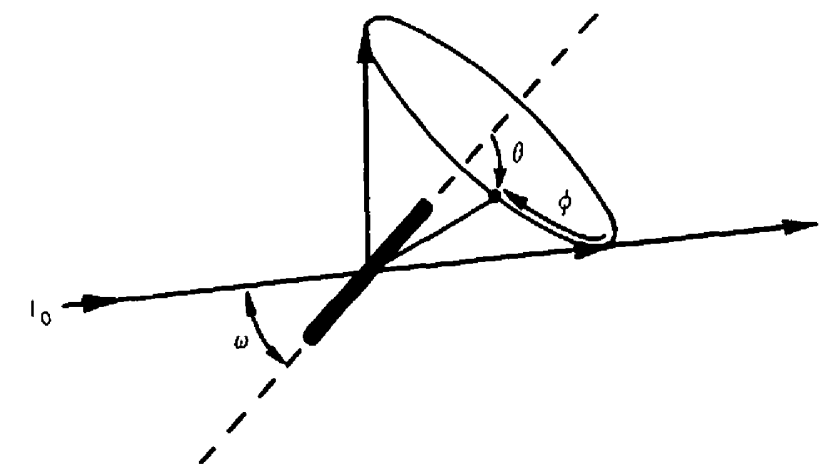

Fig. 1. Preferred scatter by a rod-shaped particle. The rod makes an angle $\omega$ with the incident beam $I_{0}$. The scattered intensity varies with the azimuth $\phi$ about the rod as axis, but for a given $\phi$ it is a maximum for colatitude $\theta=\omega$.

\section{Theory of the method}

Since bacteria are of the same order of size as the wavelength of visible light, the light scattered by a suspension is for the most part deflected little out of the direction of the illuminating beam. Whatever the shape of the particles, the scatter from an appreciable volume of suspension is axially symmetrical about the beam when the suspension is stationary, because the particles are oriented at random, but the scatter from a single rod-shaped organism is strongly dependent on direction.

The simple theory of scattering by a long and very thin rod has been known and applied for many years (Siedentopf, 1912; Stadie, 1928). The important features are shown in Fig. 1; the scattered light is concentrated in a cone whose axis is that of the rod and one of whose gencrators is the direction of the incident beam. When the rod is normal to the beam, the cone opens out into a flat sheet. The behaviour 
of rods which are neither very thin nor very long (relative to the wavelength) is qualitatively similar, but the scattered light is less completely concentrated in the preferred directions; the degree of concentration is a measure of the length:thickness ratio. Hence, if we have a means of aligning the particles in a suspension so that their long axes are normal both to an incident light beam and to a convenient direction of observation, the intensity of the scattered light will be greater than if the particles were oriented at random, and the excess will be a measure of the axial ratio.

Partial alignment is readily achieved by allowing the suspension to flow through a tube; the particles tend to sct their long axes parallel to the length of the tube. The hydrodynamic forces acting on the particles have to compcte with the disorganizing effect of thermal motion (depending on the viscosity of the medium, the temperature, and the moment of inertia of the particles). The alignment is best near the wall of the tube, where the velocity gradient is highest; on the axis there is no alignment since there is no velocity gradient. In spite of this defect, flow through a cylindrical tube is so simple to arrange that it is much to be preforred to any more nearly ideal alternative.

$\Lambda$ estimate of the flow rate necessary to effect a useful degree of orientution can be obtuined in the following way.

We may expect the orientation to be effective if the shear is large compared with the rotational Brownian movement of the particles (expressed as mean square angular displacement per unit time). The rotational Brownian movement is given by

$$
\frac{\overline{\omega^{2}}}{\bar{t}}=\frac{2 k T P}{3 \eta \bar{V}}
$$

where $\omega$ is the angular displacement from a given direction, $t$ is time, $t$ is Boltzmann's constant, $T$ is absolute temperature, $\eta$ is the viscosity of the fluid, $V$ is the volume of the particles, and $P$ is a shape factur (implicitly involving the radius of gyration of the particles). For spheres, $P$ is 1 , and for long rods it takes smaller values (Sadron, 1953); we shall not underestimate the rotation if we take $\boldsymbol{P}=1$. Then very roughly

whence

$$
\begin{aligned}
k & =1 \cdot 4 \times 10^{-16} \mathrm{ergs} / \text { degrec Centigrade. } \\
T & =300^{\circ} \text { absolute, } \\
\eta & =0 \cdot 01 \text { poise (water) } \\
V & =10^{-12} \mathrm{ml} . \text { (baccteria) }
\end{aligned}
$$

$$
\overline{\omega^{2}} i t=2 \cdot 8 \mathrm{sec}^{-1} \text {. }
$$

Now in the laminar flow of a liquid through a cylindrical tube of diameter $d$, the velocity gradient at the wall is

$$
\frac{32 Q}{\pi d^{3}} \sec ^{-1}
$$

where $Q$ is the volume flow rate. We therefore need to make

$$
\frac{Q}{d^{3}} \gg \frac{1 \cdot 2 \cdot 8 \pi}{32}=0 \cdot 27 \text {. }
$$

If we work with a tube of diameter $0.5 \mathrm{~cm} ., Q$ should be much greater than $0.04 \mathrm{ml}$. .) 
sec. This is a very modest requirement; we can expect good orientation over most of the cross-section with a flow of about $1 \mathrm{ml} . / \mathrm{sec}$. In these circumstances the light scattered by the flowing suspension will not be unduly sensitive to changes in flowrate, which incidentally is much below the critical value for the onset of turbulence.

Both the Brownian motion and the flow through the tube are inverscly proportional to the viscosity of the suspending fluid. Changes in viscosity due to temperature variation will therefore not affect the degree of orientation. The additional direct dependence of Brownian motion on absolute temperature will give rise to a small effect, probably negligible for ordinary fluctuations in ambient temperature.

The problem of scatter by a suspension undergoing shear orientation can be treated theoretically if the particles are very thin (Wippler, 1954); it is again an empirical fact that qualitatively similar behaviour is exhibited by bacteria, in spite of their less amenable shape and size.

\section{METHOD}

The indications of the preceding section were readily realized by a simple modification to a standard Pulfrich turbidimeter. In this instrument, as normally used, the suspension under test is contained in a cylindrical cuvette immersed in a waterjacket. (The jacket prevents appreciable refraction of the light entering and leaving the cuvette and helps to suppress reflexions.) Light scattered by the suspension over a range of angles near $45^{\circ}$ to the illuminating beam is collected by a lens and compared with the lamp intensity in a prismatic eyepiece.

The drain-cock of the water-jacket was removed and a glass tube, about $5 \mathrm{~mm}$. internal diameter and $15 \mathrm{~cm}$. long, was mounted centrally in the chamber. To its lower end was fused a capillary $0.5 \mathrm{~mm}$. internal diameter and $7 \mathrm{~cm}$. long, to its upper end a three-way tap communicating with the open air and with a waterpump (Fig. 2). The tube could thus be filled from below by suction and the contents allowed to drain out under gravity. The drainage rate was about $0.4 \mathrm{ml} / \mathrm{sec}$ - $-a$ higher rate would have been preferable but for the restricted sample volume.

The volume of sample required to fill the tube was about $5 \mathrm{ml}$.; a larger volume did not seem desirable. During draining, therefore, steady flow past the illuminating beam was maintained for only a few seconds, and this was too short a time to permit visual comparison of the scattered light with the illuminant. The eyepiece was therefore replaced by two photoelectric cells working into a symmetrical current amplifier (Fig. 3). The amplifier was based on a reliable circuit due to Sowerby (1944), giving in this case a gain of about $10^{6}$. Sowerby described this circuit incidentally as part of an optical instrument, and its great virtues have in consequence gone largely unrecognized. It can often be used where something less than the full sensitivity of a photomultiplier is adequate. Its special feature is simply the use of very large photocell loads together with correspondingly large cathode loads for the input valves, which are of a standard type. The essential parts of the circuit are shown in Fig. 4. Electrical supplies were derived from a conventional transformer and rectifier system with gas discharge voltage stabilizers, connected to mains through a constant voltage transformer with harmonic filter; this transformer also supplied the photometer lamp (Messrs Advance Components, I.td., Hainault, Essex. The more usual simpler type of transformer giving merely a constant r.m.s. voltage was found to be less effective, possibly because of its poor wave form). 
The zero indicator was a $200 \mu \mathrm{A}$. meter (M, Fig. 4) with two sensitivity controls, one continuous $\left(\mathrm{R}_{12}\right)$ and one a four-position switch $(S)$ giving accurately factors of $\times 1, \times \frac{2}{5}, \times \frac{1}{10}, \times \frac{1}{25}$. The shunt and series resistors associated with the switch were carefully adjusted so that changing the switch position did not alter the resistance presented across the cathodes of $V_{5}$.

The fine and coarse adjustments $P_{1}, P_{2}$ (Fig. 4) provide for balancing the amplifier so that the meter reading is zero with no light incident on either photocell.

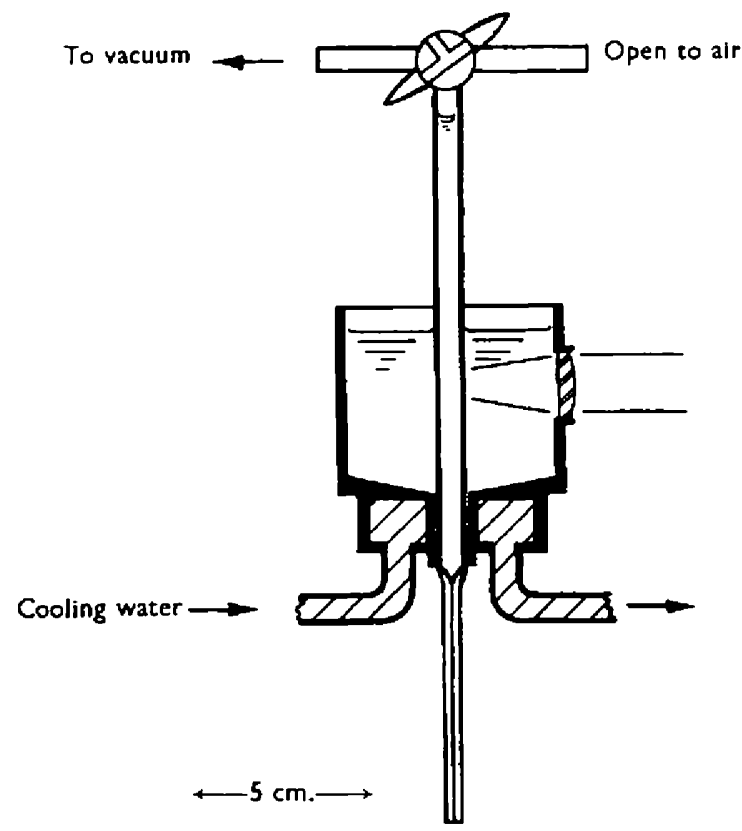

Fig. 2. Sample tube mounted in chamber of standard Pulfrich photometer (scetional elevation). Lenses for admission of light and observation are mounted in the chamber wall. Cooling water may be passed through a separate duct in the base.

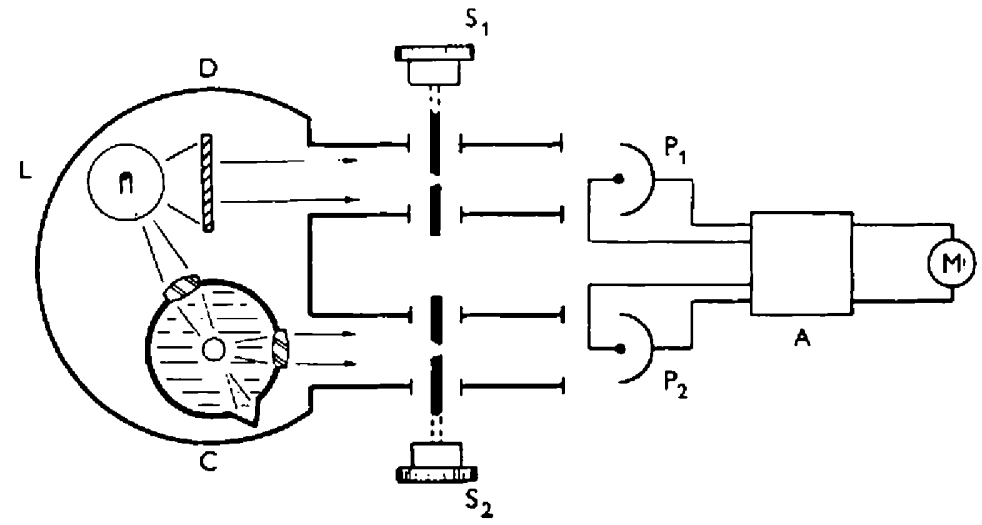

Fig. 3. Modiffed Pulfrich photometer, plan (schematic). The sample tube is seen in transverse section at the centre of the chamber $C$. $L$, lamp; $D$, diffusing screen; $S_{2}, S_{2}$, calibrated stops; $P_{1}, P_{2}$, photocells; $A$, amplifier; $M$, microammeter. 
In using the apparatus it was found very necessary to guard against incrustations and bubbles attached to the inner wall of the tube. As a washing liquid and for blank determinations we used normal saline with small additions of phosphate, formalin and an anionic detergent (Norris \& Powell, 1961). The detergent promoted foaming, but the bubbles formed did not adhere to the wall. Whenever possible, i.e. when samples of a growing culture had not to be kept at constant temperature, the formation of bubbles of dissolved gas was discouraged by cooling the chamber slightly with tap-water (Fig. 2). As usual in turbidimetric work, care had to be taken to exclude foreign particles, especially cotton wool; washing and diluting fluids were filtered twice through the same paper before use.

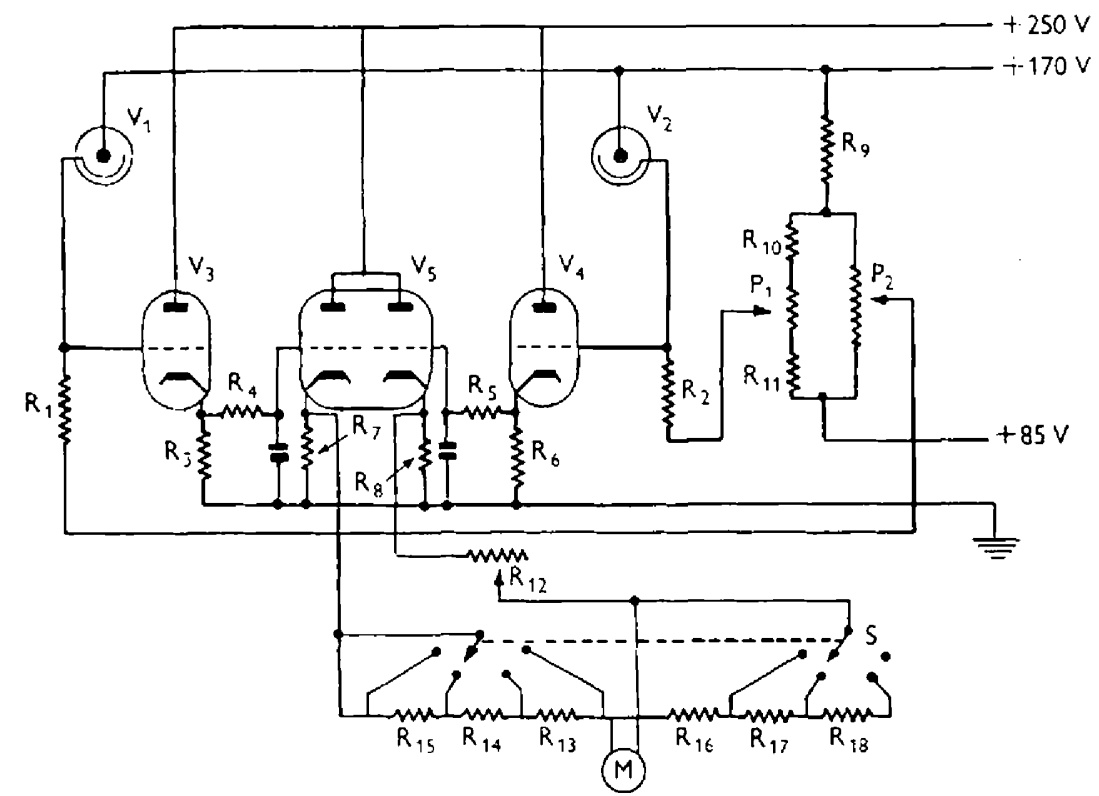

Fig. 4. Circuit diagram of amplifier, omitting power supplies. $V_{1}, V_{2}$, phetceells (Cinematelevision type VA 17T); $V_{3}, V_{4}$, type $6 Q 7$ (diodes strapped to earth); $V_{5}$, typc $12 \Lambda T 7$; $\mathbf{R}_{1}, \mathbf{R}_{2}, 200 \mathrm{M} \Omega ; \mathbf{R}_{3}, \mathbf{R}_{4}, \mathbf{R}_{5}, \mathbf{R}_{5}, 1 \mathrm{M} \Omega ; \mathbf{R}_{7}, \mathbf{R}_{\mathrm{k}}, 15 \mathrm{k} \Omega ; \mathbf{R}_{9}, 68 \mathrm{k} \Omega ; \mathbf{R}_{10}, \mathbf{R}_{11}, 10 \mathrm{k} \Omega ; \mathbf{R}_{\mathrm{t}}$, $2.5 \mathrm{k} \Omega$ variable; $P_{1}, 2 \mathrm{k} \Omega$ potentiometer; $P_{2}, 20 \mathrm{k} \Omega$ potentiometer; $C_{1}, C_{2}, 0.1 \mu F . ; R_{13}$, $164.0 \Omega ; R_{16}, 82.0 \Omega ; R_{19}, 16.0 \Omega ; R_{18}, 11.4 \Omega ; R_{17}, 18.9 \Omega ; K_{18}, 152.0 \Omega ; M, 200 \mu A$., $270 \Omega ;$ S, 2-pole, 4-way switch.

The apparatus is used in the following way.

A blank determination of parasitic scattered light is first made by filling the sample tube with saline. The calibrated stop $S_{2}$ (Fig. 3) is opened to its fullest extent. Then the stop $S_{1}$ is adjusted to give the null point on the meter $M$. The reading $(B)$ of $S_{1}$ is recorded. The saline is sucked out; the tube is washed through three times with small quantities (c. $0.5 \mathrm{ml}$.) of the suspension to be tested, and then filled slowly with it (to avoid bubble formation through too much reduction of pressure). By means of the sensitivity controls $\left(R_{12}, S\right.$, Fig. 4) the meter reading is brought to exactly $100 \mu \mathrm{A}$.; suspensions of long organisms give a constant reading only after 1-2 min. at rest. The reading then corresponds to the light intensity scattered by the suspension itself. The meter is brought to zero again by opening $S_{1}$; the new reading $(T)$ of $S_{1}$ is noted. (The difference $T-B$ gives a useful comparative 
measure of concentration for suspensions of similar organisms.) The threc-way tap is then turned to admit air above the suspension; as it drains out, the meter reading rises within 1 or 2 sec. to a maximum value. The foregoing procedure provides that the maximum reading in microamperes is the percentage increase in the scattered light caused by shear orientation. If the increase is small, the operation is repeated, and before the suspension is allowed to drain, the meter sensitivity is increased to a higher value with the switch $S$ (Fig. 4). The new maximum reading then exceeds the true increase by an exactly known factor. In any case it is desirable to repeat the observation two or three times. $A$ defect of the apparatus is the change in flow-rate as the liquid level falls. Fairly reproducible readings are obtained by working only with the meniscus between roughly fixed limits, e.g. the uppermost $3-4 \mathrm{~cm}$. of the tube.

To avoid mechanical damage, the meter sensitivity is set to its extreme minimum (both controls) during emptying and filling of the sample tube.

\section{Some representalive results}

This apparatus has been used successfully in collaboration with Dr J. IR. Postgate for following the growth of synchronized cultures, but our purpose here is to give no more than an indication of its scope and possibilities.

Table 1. Fxamples of increased light scattering by flowing suspensions in the modified Pulfrich turbidimeter

$\begin{array}{lc}\text { Polystyrene spheres ( } \mathbf{1 . 8} \mu \text { diam.) } & \begin{array}{c}\text { Shear effect } \\ \text { (\% increase) }\end{array} \\ \begin{array}{l}\text { Chromolacterium prodigiosum } \\ \text { (very small resting cells) }\end{array} & 0 \\ \begin{array}{c}\text { Aerobacter aerogenes (from steady } \\ \text { growth in continuous culture) }\end{array} & 5 \\ \text { Human erythrocytes in saline } & 28\end{array}$

A general impression of the magnitudes involved is given by Table 1. Human erythrocytes are included as an example to show that the application can be extended to non-sphericul particles other than rods; in a number of industries there are control problems to which the principle might be applicd: cosmetics, paint, explosives. For convenience, we use the phrase 'shear effect' to mean the porcentage increase in light scatter during flow, as measured in the apparatus we have described.

If the concentration and thickness (in the direction of the illuminating bcam) of a sample of a suspension are not too lare, the individual particles scatter independently of one another and their effects are additive. Van de IIulst (1957) suggests as a working rule that this is true up to an optical density of about 0.1. Within this range we should expect the shearing cffect (which is a ratio of turbidities) to be independent of concentration, and this is in fact nearly true up to appreciably higher densities. The useful concentration range of the apparatus we have described is, for bacteria, from about $2 \times 10^{7}$ to $2 \times 10^{9}$ per $\mathrm{ml}$., depending on the kind of organisms, but for synchrony work the concentration chosen should be as low as is convenient. The shearing effect is then influenecd by changes in shape but not in number. Table 2 illustrates the dependence of slicar effect on concentration. 
The shear effect is not greatly influenced by the colour of the light. At high concentrations violet light produces a detectably smaller effect than green or red (presumably because the optical density is higher for the shorter wavelength), but there seems to be no reason for using filtered light in ordinary practice.

Table 2. Dependence of shear effect on concentration. Acrobacter aerogenes in saline. Mean length of organisms: $5 \cdot 47 \mu ;$ mean breadth: $0 \cdot 50 \mu ;$ ratio, $R, 10 \cdot 8$

$\begin{array}{ccc}\begin{array}{c}\text { Concentration, } \\ \text { No. } / \mathrm{ml} . \times 10^{-0}\end{array} & \begin{array}{c}\text { Turbidity } \\ \text { (arbitrary units) }\end{array} & \begin{array}{c}\text { Shear effect } \\ \text { (\% increase) }\end{array} \\ 2.2 & 50.9 & 19.6 \\ 0.75 & 20.8 & 26.5 \\ 0.25 & 6.1 & 33.9 \\ 0.083 & 1.9 & 35.9\end{array}$

Suspensions of bacteria usually contain organisms having a considerable dispersion of size, and the shearing effect observed is a weighted mean of the various effects duc to the individual particles. Suppose we write $T_{+}$for the scattered intensity from a given suspension at rest and $T_{\text {s }}$ for the scattered intensity from the flowing suspension, then what we have called the shear effect is

$$
100\left(T_{s} / T_{r}-1\right) \text {. }
$$

It is possible to write down a formal expression for $T_{k} / T_{r}$ as a weighted mean of contributions from the different particles, but it is of little practical value because the measured seatter depends in a very complex way on both general optical laws and on the geometry of the apparatus. However, the way in which shear effects combine can readily be illustrated. Suppose we have two suspensions, $a$ and $b$, giving scattered intensities $T_{r, a}, T_{r, b}$ (stationary) and $T_{s, a}, T_{s, b}$ (flowing); then in a mixture with volume fractions $\alpha$ and $\beta$ of $a$ and $b$, respectively, we have

$$
\begin{aligned}
& T_{r}=\alpha T_{r, a}+\beta T_{r, b}, \\
& T_{s}=\alpha T_{s, a}+\beta T_{s, b},
\end{aligned}
$$

provided the concentrations are low enough to be directly proportional to turbidity in each suspension severally. Then

$$
\frac{T_{s}}{T_{r}}=\frac{\alpha T_{r, a}}{\alpha T_{r, a}+\beta T_{r, b}} \frac{T_{s, a}}{T_{r, a}}+\frac{\beta T_{r, b}}{\alpha T_{r, a}+\beta T_{r, b}} \frac{T_{s, b}}{T_{r,}}
$$

writing $C, C_{a}, C_{\mathrm{b}}$ for the shear effects (so that $C=100\left(T_{\mathrm{s}} / T_{\mathrm{r}}-1\right)$, etc.),

$$
C=\frac{\alpha T_{r, a}}{\alpha T_{r, a}+\beta \bar{T}_{r, b}} C_{a}+\frac{\beta T_{r, b}}{\alpha T_{r, a}+\beta T_{r, b}}-C_{b} .
$$

The truth of this equation was tested in a number of experiments by making up mixtures of two radically different suspensions, and comparing the observed and calculated shear effects. The agreement was uniformly satisfactory; Fig. 5 gives an extreme example.

Figure 6 is a conspectus of the association between shear effect and the ratio, $R$, of the mean length to mean breadth of the organisms in the suspensions concerned. It covers a wide variety of suspensions, including several samples from continuous cultures in which growth restriction had produced exaggerated forms of size-distribution. The correlation is perhaps less perfect than might at first be expected; 
the estimates of $R$ were based each on the measurcments of rather few organismsnever more than 40 -and are subject to appreciable errors, both statistical and experimental. It has in any case to be remembered that suspensions having the same value of $\boldsymbol{K}$ will give different shear effects if their size distributions are different. Morcover, the shear effect is not a function, evell in homogeneous suspensions, of the shape only, but depends also on the absolute size and refractive index, and is affected by non-uniformity within the particles (c.g. organisms in a state of incipient fission). Spores of Bacillus alvei provide an example; the spores are enclosed in spindle-shaped envelopes with long pointed ends and of low optical density. They are represented in Fig. 6 by two entries; for the one, $R$ is taken to be
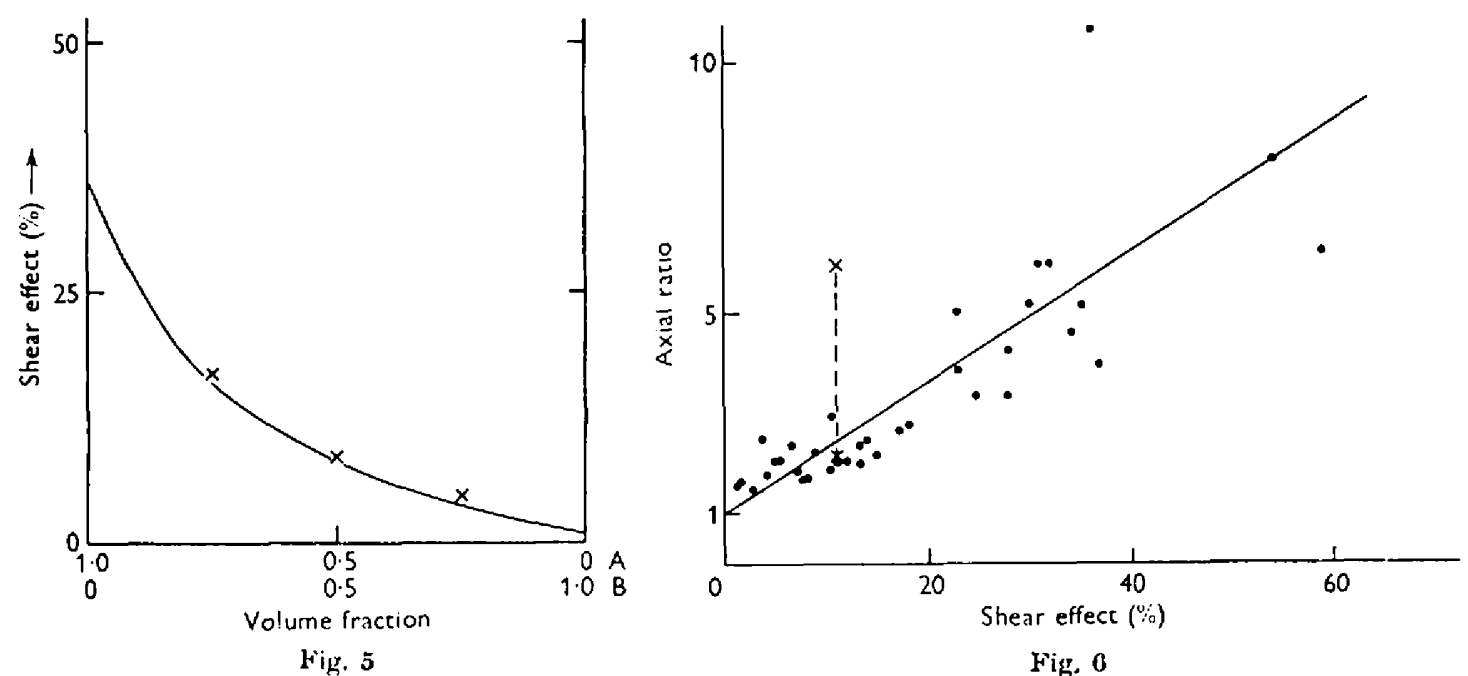

Fig. 0

Fig. 5. Shear effect in mixtures. Suspension A: Aerobarter aerogenes, 8.3 $\times 10^{7} / \mathrm{ml}$; relative turbidity, $1 \cdot 7$; shear effect $36.0 \%$. Suspension B: Bacillus subtilis spores, $1.23 \times 10^{8} / \mathrm{ml}$; relative turbidity, 6.9 ; shear effect $1.1 \%$. Continuous curve, calculated; crosses, experiniental values.

Fig. 6. Summary diagram of relation between shear effect and axial ratio; various suspensions. Bacillus alvei spores are represented by two entries joined by a dotted line. The larger axial ratio is that of the envelope, the smaller that of the spore itself. The solid line is the regression of axial ratio on shear effect (assumed linear).

the ratio of the extreme dimensions of the spindle, for the other, of the dense central mass only. By the one standard, the shear effect is unusually low; by the other, unusually high.

\section{Size fluctuation in synchronous groweth}

Stárka \& Koza (1959) described an experiment in which the growth of a synchronized culture was followed photometrically as well as by counting the viable organisms. They found that in their apparatus the turbidity increased in the same stepwise manner as the number concentration; during periods of growth without fission the turbidity did not change in spite of the increasing size of the organisms; during periods of rapid fission it followed the increase in number. This rather surprising result is due, we think, to the geometry of the instrument used-the Pulfrich turbidimeter with scattered light collected at $45^{\circ}$ to the illuminating beam. 
An increase in particle size brings about a greater concentration of the scattered light in directions close to the incident beam, and a reduction in intensity in remote directions; there will be a range of angles within which the intensity changes little. This condition seems to apply here; to some extent Stárka \& Koza's result is a happy accident. We have carried out an experiment of the same kind, using a similar turbidimeter and a synchronous culture of Aerobacter aerogenes obtained by fractional filtration according to the method of Abbo \& Pardec (1960). The stepwise change in turbidity was well-marked though less sharp than in Stárka \& Koza's experiment. Similar measurcments carried out by Abbo \& Pardee with a different form of photometer showed much less clearly marked steps.

In well-developed synchronous growth the number concentration very nearly doubles during the fission phase, and the above example shows that in favourable cases the turbidity may do the same. The changes in mean size which occur, however, are not only smaller but less rapid, as is shown qualitatively by the following hypothetical illustration.

Suppose we have a suspension of organisms all of which have just divided at time $t=0$, such as might be obtained by fractional filtration of a growing culture. Assume (i) that the organisms all have the same length $x_{0}$ initially; (ii) that the diameter of the organism is constant but the length $x$ (hence the volume) increases exponentially at the same rate $v$ as the steady growth rate of the culture: $x=x_{0} e^{v t}$; (iii) that the distribution $f(\tau)$ of generation times $\tau$ is so narrow that almost all the organisms have divided before any of the first-generation progeny has done so. Then if there are initially $N$ organisms, the actual fission rate $d N / d t$ will be $N f(t)$ during this period. At time $t$ there will be

$$
2 N \int_{0}^{t} f(\tau) d \tau=\operatorname{say} 2 N F_{+}(t)
$$

first-generation progeny, of mean length $\frac{1}{2} x_{0} e^{\nu t}$, and

$$
N \int_{i}^{\infty} f(\tau) d \tau=N\left\{1-F_{+}(t)\right\}
$$

original organisms still undivided, of length $x_{0} e^{\nu t}$. The mean length is thus

$$
\begin{aligned}
& \bar{x}=\frac{N\left\{1-F_{+}(t)\right\} x_{0} e^{\nu t}+N F_{+}(t) x_{0} e^{\nu t}}{N\left\{1-F_{+}(t)\right\}+2 N \bar{N} F_{+}(t)} \\
& \bar{x}=\frac{e^{\nu t}}{1+F_{+}(t)} .
\end{aligned}
$$

For the purpose of the illustration we may take $f(\tau)$ to be a Pearson Type III distribution

$$
f(\tau) \equiv \frac{\tau^{y-1} e^{-g / m}}{m^{g} \Gamma(g)^{-}}
$$

The value of the parameter $m$ affects only the time-scale-we may take $m=1$; $g$ is a measure of the dispersion, and a representative value of 35 is suggested by actual measurements (Powell, 1958). Then $\nu=0.0200 \mathrm{~min}^{-1}$. Hence $\bar{x}$ may be calculated as a function of $t$. The result is shown in Fig. 7 ; in this case the maximum mean length is only $1 \cdot 6 x_{0}$, not $2 x_{0}$; the fall in $\bar{x}$ occurs during the earlier stages of the fission phase and is less striking than the increase in numbers. (This simple reasoning no longer applies after the first-generation progeny have begun to divide.) 
In real cultures, there is always an appreciable dispersion of size in the initial population, and it is not known that individual organisms grow at the same constant rate. Nevertheless, the example will serve as a warning not to cxpect a simple geometrical relation between the curves representing changes in mean size and changes in number. In fact the example agrees quite well with such experimental figures as have been obtained; we are indebted to Dr J. R. Postgate for providing data for Fig. 8.

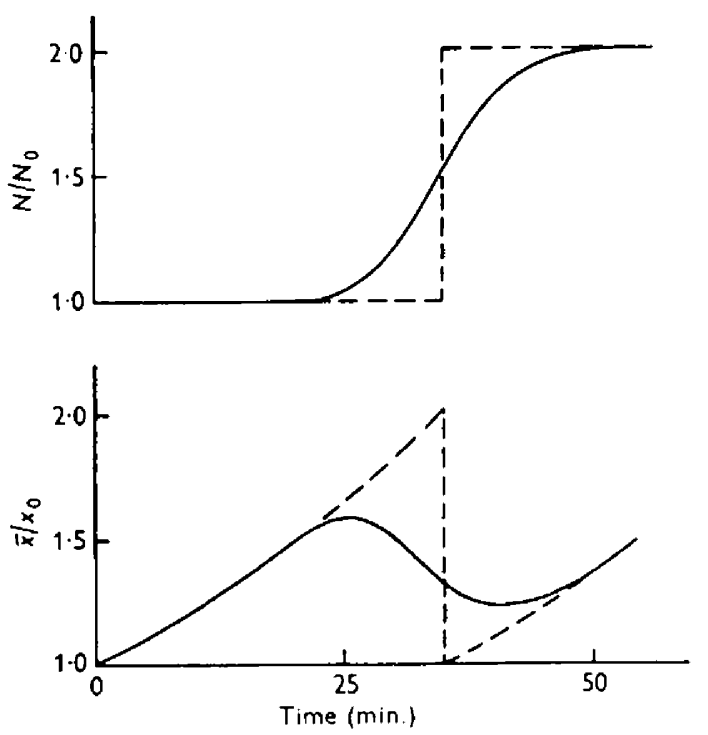

Fig, 7

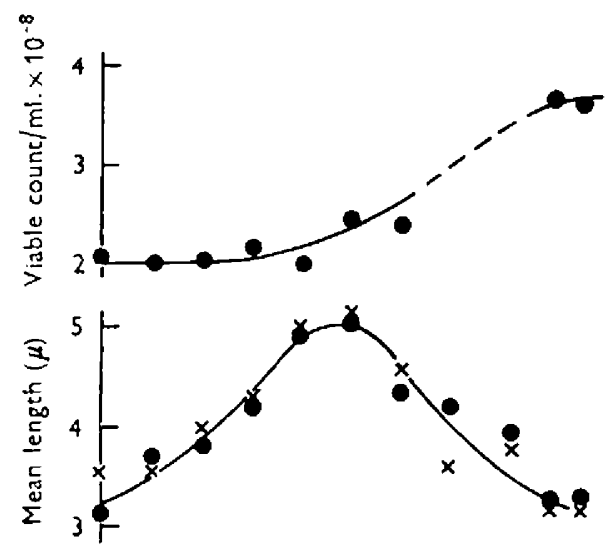

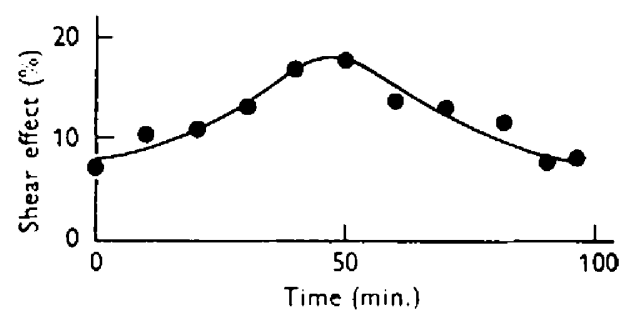

Fig. 8

Fig. 7. Illustrating variation of mean length of organisms in synchronous growth. Upper curves: increase in numbers $(N)$; lower curves: changes in mean length $(\bar{x})$. The dotted curves show the result to be expected in the absence of any dispersion of generation time. Calculated for mean generation time $35 \mathrm{~min}$., standard deviation $5.9 \mathrm{~min}$.

Fig. 8. Farly development of a culture of Aerobacter aerogenes synchronized by repeated starvation. Cpper curve: viable count. Lower curve: shear effect. Middle curve: crosses--measured mean length; circles-mean length estimated from shear effect (J. R. Postgrate).

\section{Further development}

The apparatus we have described could not well be simpler, but it is in many cases quite adequate for checking the development of supposedly synchronized cultures. Its sensitivity, reproducibility, and range of application could readily be. improved by attention to some obvious defects, particularly the poor control of flow-rate and temperature, and the large and impcrfectly compensated blank reading due to reflexions at glass-liquid interfaces.

The sensitivity, in particular, could in principle be increased by illuminating the sample with polarized light, but rough experiments suggest that the advantage would be slight. A particular suspension, for example, giving a shear effect of 
$28.1 \%$ in natural light gave in polarized light values of 26.2 and $30.7 \%$ when the electric vector was respectively perpendicular and parallel to the axis of the sample tube. As we constructed it, the apparatus is not quite accurate enough for work on organisms of low axial ratio such as spores $(R=1 \cdot 5$, e.g. $)$ which give a shear effect of only 1-3\%. A more accurate instrument would make it worth while to investigate in more detail the dependence of shear effect on dispersion of size. It is clear that the arithmetic means we have used are not really appropriate to the problem, and some form of weighted mean might be found, much more closely correlated with the shear effect.

It should also be possible, by a refinement of the electronic detector, to measure the dispersion as well as the mean of an axial ratio. When the flow of a suspension is suddenly stopped, the shear orientation exhibits a relaxation time which is surprisingly long - frequently many seconds elapse before the turbidity reverts to its stationary value (cf. the estimate of rotational Brownian motion on p. 491 above). Consider two suspensions, the first of particles of moderate axial ratio, the second of a mixture of spheres with very long particles, adjusted to have the same shear effect as the first. Then the relaxation time of the second will be the same as if it contained the long rods only, since the spheres contribute nothing to the shear effect. The relaxation time of the homogeneous suspension will be the shorter. Hence if we can estimate the rate of decay of the shear effect (i.e. of the excess scattered light) when the flow is stopped, the result can be used in combination with the magnitude of the effect as a measure of the dispersion of axial ratio.

We are indebted to W. C. Wright for constructing the photoelectric detector, and to P. F. Errington for making many measurements. One of us (P. J. S.) carried out this work while acting as a Vacation Consultant from the College of Advanced Technology, Birmingham.

\section{REFERENCES}

Ambo, F. \& PARDeE, A. B. (1960). Synthesis of macromolecules in synchronously dividing bacteria. Biochim. biophys. Acta, 39, 478.

vaN DE Hulst, H. C. (1957). Light Scattering by Small Particles. New York: John Wiley and Sons, Inc.

NoRRIs, K. P. \& Powell, E. O. (1961). Improvements in determining total counts of bacteria. J. R. micr. Soc. 80, 107.

Powels, E. O. (1958). An outline of the pattern of bacterial generation times. J. gen. Microbiol. 18, 382.

Poweld, E. O. (1962). Photometric methods in bacteriology. J. Sci. Fd Agric. (In the Press.)

Sadron, C. (1953). Methods of determining the form and dimensions of particles in solution: A critical survey. In Progress in Biophysics, 3, 237. Ed. J. A. V. Butler \& J. T. Randall. London: The Pergamon Press, Ltd.

Siedentopf, H. (1912). Utber ultramikroskopische Abbildung linearer Objekte. $Z$. wiss. Mikr. 29, 1.

Sowerby, J. McG. (1944). A photoelectric photometer for measuring the light scattered by the surface of a transparent material. J. sci. Instrum. 21, 42.

Stanie, F. (1928). Probleme der Brownschen Molekularbewegung. Ann. Phys. Lpz. 86, 751.

StírkA, J. \& KozA, J. (1959). Nephelometric determination of cell count in synchronously dividing cultures of bacteria. Biochim. biophys. Acta, 32, 261.

Wippler, C. (1954). Étude théorique de la diffusion de la lumière par les sols de batonnets orientés. J. Chim. phys. 51, 122. 